\title{
A Novel Approach for Thunderstorm and Lightning Detection System
}

\author{
Rasika Kalbende ${ }^{1}$, Nitin Shelke ${ }^{2}$ \\ ${ }^{1,2}$ Computer Science Department, G.H.Raisoni College of Engineering \& Management, Amravati, India
}

\begin{abstract}
Thunderstorm and lightning is a sudden electrical expulsion manifested by a blaze of lightening with a muffled sound. It is one of the most spectacular mesoscale weather phenomena in the atmosphere which occurs seasonally. Every thunderstorm produce lightening, this kills more people every year than tornadoes, and prediction of thunderstorms is said to be the most complicated task in weather forecasting, due to its limited spatial and temporal extension either dynamically or physically. Various researches are been carried on for forecasting of this severe to reduce damage. Many of the researchers proposed various methodologies like STP model, MOM model, CG model, LM model, QKP model, DBD model and so on for the detection, but neither of them could provide an accurate prediction.The proposed system is to gather the satellite images obtained from dataset in order to predict whether the cloud images produces thunderstorms or not. The proposed system adopted clustering and wavelet transform techniques for thunderstorms and lightning detection using image processing and data mining. The proposed system improves the prediction rate to a greater extent, on the basis of some statistical analysis.
\end{abstract}

Keywords: K-medoid Clustering, Wavelet Transform, ANN

\section{Introduction}

Computers are widely utilized in today's weather forecasting as a powerful tool to leverage an enormous amount of data. Yet, despite the availability of such data, current techniques often fall short of producing reliable detailed storm forecasts. Each year severe thunderstorms cause significant damage and loss of life, some of which could be avoided if better forecasts were available.

\section{Thunderstorm}

Thunderstorm is a vicious, climatic disturbance that is associated with heavy rains, lightening, thunders, thick clouds and gusty surface winds. Thunderstorms take place when a layer of warm and moist air rises to a larger extent, and updrafts to the cooler regions of the atmosphere. The updraft that contains moisture condenses in order to form massive cumulonimbus clouds and eventually leads to the development of precipitation. Columns of frozen air then sink earthward, striking the ground with strong downdrafts and horizontal winds. Meanwhile, electrical charges mount upon cloud particles and causes lightning. This further heats the air in a violent manner by which shock waves are produced, resulting in thunder. Usually, thunderstorms have the spatial area for a few with a life span less than an hour. However, multi-cell thunderstorms have a life span of several hours and may travel over a few hundreds of kilometers. Throughout the world it is estimated that 16 million thunderstorms occur each year, and at any given moment, there are roughly 2,000 thunderstorms in progress. There are about 100,000 thunderstorms each year in the U.S. alone. About $10 \%$ of these reach severe levels. Under the right conditions, rainfall from thunderstorms causes flash flooding, killing more people each year than hurricanes, tornadoes or lightning Cloud to ground lightning frequently occurs as part of the thunderstorm phenomena, which on severity becomes hazardous to the property, wildlife and population across the globe to a major extent. One of the most significant lightning hazards is to the wildfires, as they can even ignite the ground surfaces. Wildfires can devastate vegetation and the biodiversity of an ecosystem. Cloud to ground lightning frequently occurs as part of the thunderstorm phenomena, which on severity becomes hazardous to the property, wildlife and population across the globe to a major extent. Following figure shows how thunderstorm developed under three stages i.e, Life cycle of thunderstorm.
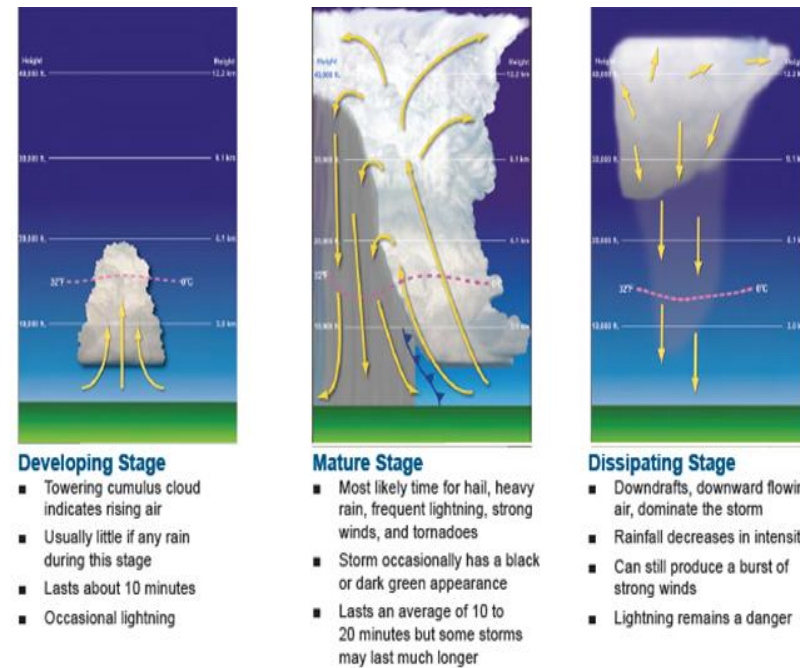

Dissipating Stage

- Downdrafts, downward flowing air, dominate the stor

- Rainfall decreases in intensity

- Can still produce a burst of strong winds

- Lightning remains a danger

Figure 1: Thunderstorm Life Cycle

\section{Lightning}

The rising air in a thunderstorm cloud causes various types of frozen precipitation to form within the cloud. Included in these precipitation types are very small ice crystals and much larger pellets of snow and ice. The smaller ice crystals are carried upward toward the top of the clouds by the rising air while the heavier and denser pellets are either suspended by the rising air or start falling toward the ground. Collisions occur between the ice crystals and the pellets, and these collisions serve as the charging mechanism of the thunderstorm. The small ice crystals become positively charged while the pellets become negatively charged. As a result, the top of the cloud becomes positively charged and the middle to lower part of the storm becomes negatively 


\section{International Journal of Science and Research (IJSR) \\ ISSN (Online): 2319-7064}

Index Copernicus Value (2015): 78.96 | Impact Factor (2015): 6.391

charged. At the same time, the ground underneath the cloud becomes charged oppositely of the charges directly overhead .When the charge difference between the ground and the cloud becomes too large, a conductive channel of air develops between the cloud and the ground, and a small amount of charge (step leader) starts moving toward the ground. When it nears the ground and upward leader of opposite charge connects with the step leader. At the instant this connection is made, a powerful discharge occurs between the cloud and the ground. We see this discharge as a bright visible flash of lightning. Following figure shows lightning image.

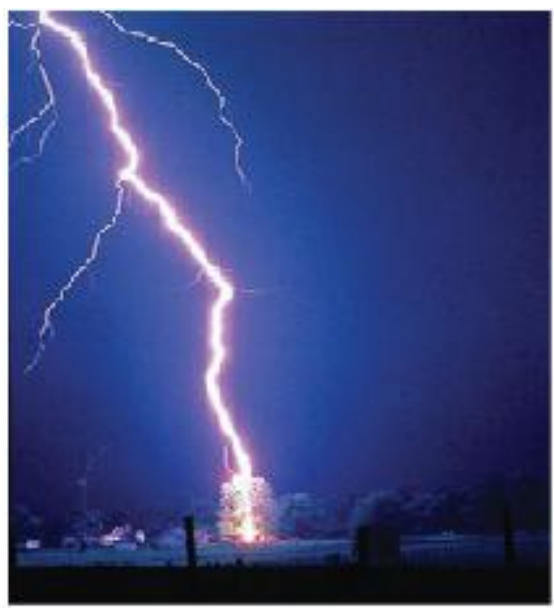

Figure 2: Lightning Image

\section{Proposed Work}

The goal of this research is to scrutinize the satellite images obtained from Indian Meteorological Department, in order to predict whether the cloud images produces thunderstorms or not and find out whether lightning touches to the ground or not.

Initially, the original satellite image of clouds is taken as the input image for the experimentation. As the input image is a satellite image, it may restrain with different type of noises such as striping noise, speckle noise, blurs and so on which are ought to be removed. It may also contains various textures such as water bodies, forests, grass, asphalt, barren lands, concrete, clouds and so on. These textures are to be estranged to acquire the image of interest so that the other texture does not have an effect on the precise forecasting of thunderstorms. If the satellite image containing such types of noises and textures are analyzed, the result obtained may deviate from original value. So, the input image must be segmented. Clustering is an efficient technique to segment the input image into several clusters based on similarity measure, here Euclidean distance is used as one of the similarity metric. In the present research, k-medoids clustering is adopted for segmenting the image. Here, Segmentation is performed to image by based on various color factors because colors possess wavelength values. The image containing relatively similar wavelength values are grouped into different clusters. Here, the Haar wavelet transform is adopted for the further analysis

As satellite image is an RGB image, Haar wavelet transform automatically converts RGB image into gray scale image and further de noise the image and present it in one dimension.

\section{Detection of Thunderstorm}

Following figure shows how to find out thunderstorm result by using two algorithm i.e K-mediods and harr wavelet transform. Where K-medoids is used for clustering purpose and harr wavelet is use to generate wavelet image as well as it is use to find out the wavelength range.

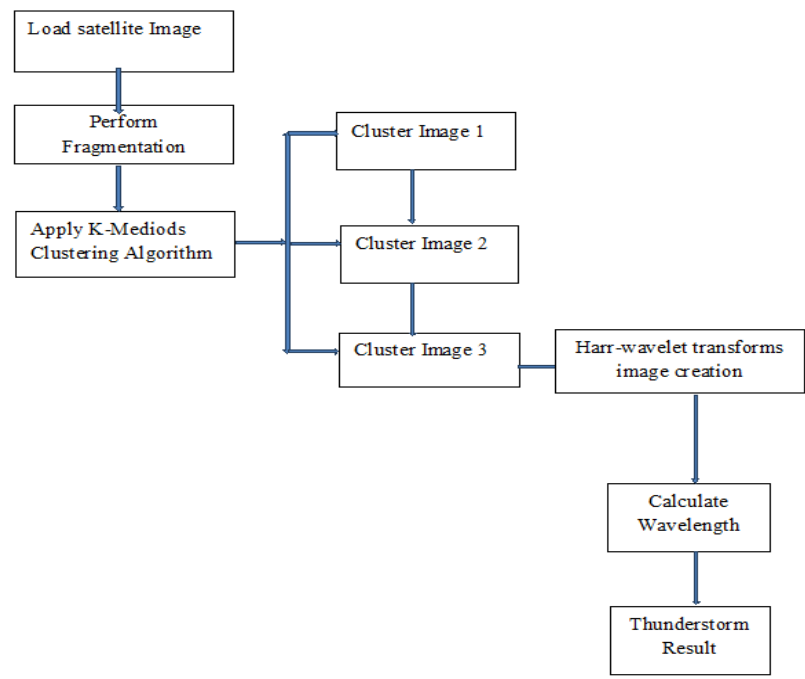

Figure 3: Flow Chart of Thunderstorm Detection

Steps for Detection of thunderstorm

Steps 1 - Gather the satellite images from Indian metrological department and load a image in order to predict whether the cloud images produce Thunderstorm or not.

Step 2 - Perform fragmentation in order to detect objects or divide the image into regions which can be considered homogeneous according to a given criterion, such as color, motion, texture.

Step 3 - Apply k-mediod algorithm, and create three clusters i.e. cluster image1 cluster image2, cluster image3.

Step 4 - Using cluster image 3, apply harr-wavelet algorithm, which convert RGB image into gray scale image and de noise the image and present it in one dimension.

Step 5 - Calculate wavelength, Based on wavelength value, it determine whether input image having thunderstorm or not. As wavelength range is between $250-350 \mathrm{~nm}$. Then result is thunderstorm.

\section{Detection of Lightning Position from Ground}

Following figure shows flow chart of detection of lightning position from ground by scanning input image pixel by pixel depending on pixel result get known. 
International Journal of Science and Research (IJSR)

ISSN (Online): 2319-7064

Index Copernicus Value (2015): 78.96 | Impact Factor (2015): 6.391

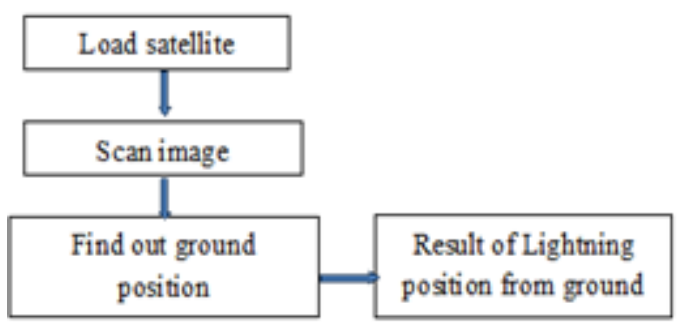

Figure 4: Flow Chart of Detection of Lightning Position from Ground

Steps for Detection of Lightning Position from Ground

Step 1 - Gather the satellite images from Indian metrological department and load a image in order to find out lightning position from ground.

Step 2 - Remove background of lightning images so that lightning part is clear visualized.

Step 3 - Scan image pixel by pixel tin order to find out ground position, and if pixel value is greater than $85 \mathrm{~nm}$ then result should be "lightning touches to the ground" otherwise "lightning not touches to the ground".

\section{Result Analysis}

In proposed system, the satellite images obtained from Indian Meteorological Department is analyzed to identify the presence of thunderstorms within the clouds. On analysis of these satellite images a square root balance sparsity norm threshold value is computed and is established to be in between an optimal range of 9 - 11.As satellite image is a visible spectrum, its wavelength value always lies in the range of $350 \mathrm{~nm}-450 \mathrm{~nm}$. Based on this criterion, the wavelength range for the feature extracted images is tested and on observation of these results, a range of $350 \mathrm{~nm}$ $450 \mathrm{~nm}$ is established for the clouds containing thunderstorms. The main goal of the present research is to detect the thunderstorms as accurate as possible. In order to compute accuracy for the present research TP, TN, FP, FN values are to be computed. The true positive (TP) specifies the positive tuples that were correctly labeled. The true negative (TN) specifies the negative tuples that were correctly labeled. The false positive (FP) specifies the negative tuples that are incorrectly labeled. The false negative (FN) specifies the positive tuples that are incorrectly labeled. The four basic performance measures i.e. sensitivity, specificity, accuracy and precision are computed for the present research in order to test how well the proposed system is working and the computations are done by using following equations .

Sensitivity $=\mathrm{TP} /(\mathrm{TP}+\mathrm{FN})$

Specificity $=\mathrm{TN} /(\mathrm{FP}+\mathrm{TN})$

Accuracy $=(\mathrm{TP}+\mathrm{TN}) /(\mathrm{TP}+\mathrm{FP}+\mathrm{FN}+\mathrm{TN})$

Precision $=\mathrm{TP} /(\mathrm{TP}+\mathrm{FP})$

Table 1: Performance Measure Factors

\begin{tabular}{cc}
\hline \hline Performance measure & Percentage (\%) \\
\hline Sensitivity & 92.10 \\
Specificity & 85.18 \\
Accuracy & 89.23 \\
Precision & 89.74 \\
\hline
\end{tabular}

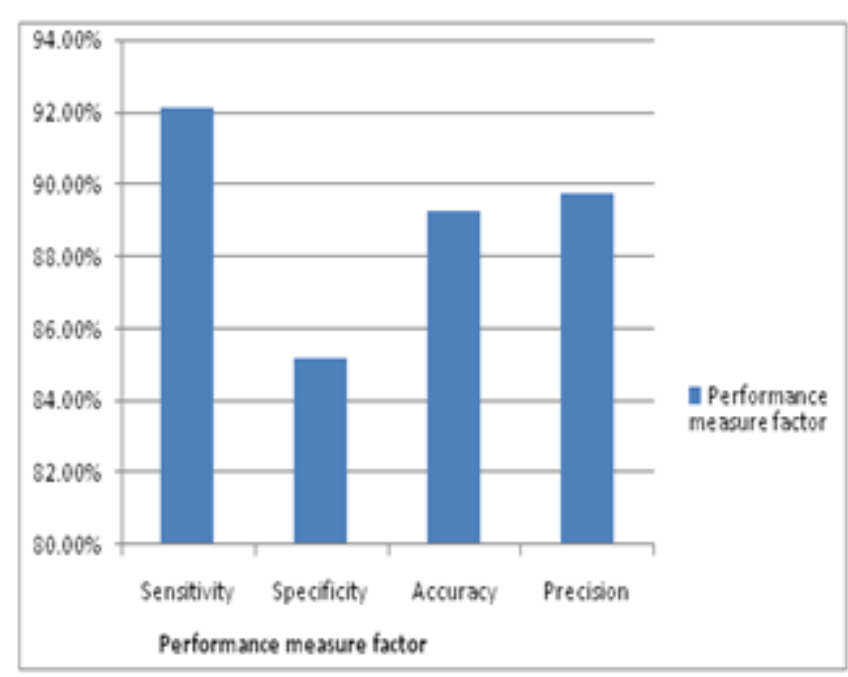

Figure 5: Graph of Performance Measure Factors

Above table performance measure factors and above figure graph of performance measure factor shows the performance of proposed system calculated in terms of parameter such as Sensitivity, Specificity, Accuracy, and Precision. There it is observed that the proposed system gives the better result for the given parameter.

Table 2: Comparison Accuracy of Proposed Model

\begin{tabular}{ll}
\hline Model & Accuracy (\%) \\
\hline STP model & 39 \\
MOM model & 42 \\
CG model & 61 \\
LM model & 76 \\
QKP model & 38 \\
DBD model & 39 \\
Proposed model & $\mathbf{8 9 . 2}$ \\
\hline
\end{tabular}

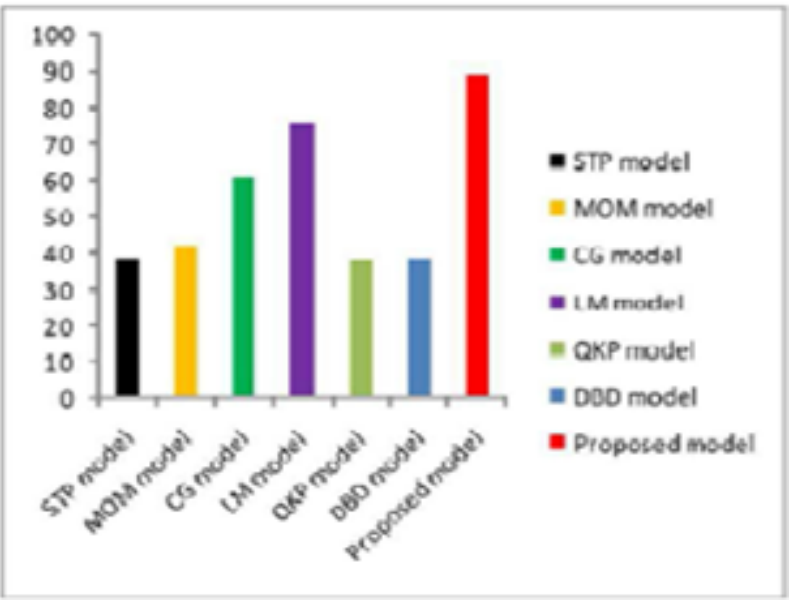

Figure 6: Comparison Graphs for Proposed Model

The proposed method is compared with previous methodologies for the prediction of thunderstorms. The comparison graph is drawn for all the algorithms and is shown in above figure. The graph clearly shows that the proposed method is outperforming when compared with the previous methodologies. 


\section{International Journal of Science and Research (IJSR) ISSN (Online): 2319-7064}

Index Copernicus Value (2015): 78.96 | Impact Factor (2015): 6.391

Table 3: Result Analysis of Thunderstorm Detection System

\begin{tabular}{|c|c|c|c|c|c|c|}
\hline $\begin{array}{l}\text { Image } \\
\text { Number }\end{array}$ & $\begin{array}{c}\text { Standard } \\
\text { Deriation( }(\theta)\end{array}$ & $\begin{array}{l}\text { Square Root } \\
\text { Balance } \\
\text { Sparsity } \\
\text { Norms } \\
\text { Thresbold } \\
\text { (t) }\end{array}$ & $\begin{array}{l}\text { Wardength } \\
\text { Factor (dSdi) }\end{array}$ & $\begin{array}{l}\text { Warelkgth } \\
(0 .)=\left(\tau^{*} d \Delta d\right)\end{array}$ & $\begin{array}{c}\text { Type of } \\
\text { Thumerstorm }\end{array}$ & $\begin{array}{l}\text { Ligtning } \\
\text { Position }\end{array}$ \\
\hline Imagel.jpg & 295 & 1096 & 30.89 & 455.29 & $\begin{array}{c}\text { Severe } \\
\text { Thanderstorm }\end{array}$ & $\begin{array}{l}\text { Touth to } \\
\text { ground }\end{array}$ \\
\hline Image2 Jpg & 2.3 & 9.88 & 39.71 & 399.64 & Theoderstorm & $\begin{array}{l}\text { Toudt to } \\
\text { ground }\end{array}$ \\
\hline Image3 Ipg & 193 & 1025 & 47.2 & 414.82 & Thanderstorm & $\begin{array}{l}\text { Touch to } \\
\text { ground }\end{array}$ \\
\hline Imaget Jpg & 273 & 1013 & 33.41 & 409.96 & Thaoderstom & $\begin{array}{l}\text { Toud to } \\
\text { groust }\end{array}$ \\
\hline Ingget Jpg & 294 & 10.5 & 31.05 & 424.94 & Thaderstorm & $\begin{array}{l}\text { Touth to } \\
\text { grond }\end{array}$ \\
\hline Lmago6 Jpg & 203 & 9.75 & 4.83 & 39.58 & Thaderstom & $\begin{array}{l}\text { Touth to } \\
\text { grond }\end{array}$ \\
\hline Image7.Jpg & 203 & 9.75 & 4.83 & 394.58 & Thaderstom & $\begin{array}{l}\text { Touth to } \\
\text { ground }\end{array}$ \\
\hline Ingas: Jpg & 199 & 1025 & 45.75 & 414.82 & Thuoderstorm & $\begin{array}{l}\text { Toud to } \\
\text { grond }\end{array}$ \\
\hline Inagee Jpg & 279 & 1038 & 3269 & 420.08 & Thuoderstorm & $\begin{array}{l}\text { Touth to } \\
\text { ground }\end{array}$ \\
\hline Image $10.5 g$ & 183 & 9.78 & 49.83 & 395.84 & Thuoderstorm & $\begin{array}{l}\text { Touch to } \\
\text { groud }\end{array}$ \\
\hline Image11.Jpg & 252 & 994 & 3617 & 402.19 & Thaderstorm & $\begin{array}{l}\text { Toud to } \\
\text { gromd }\end{array}$ \\
\hline Imaget $12 . j p g$ & 2.3 & 9.63 & 39.68 & 389.52 & Thuderstorm & $\begin{array}{l}\text { Touth to } \\
\text { ground }\end{array}$ \\
\hline Image $13 . j p g$ & 202 & 9.67 & 4507 & 391.34 & Thanderstorm & $\begin{array}{l}\text { Touch to } \\
\text { groud }\end{array}$ \\
\hline Imagt $14 . j p g$ & 242 & 9.63 & 37.68 & 399.52 & Thuoderstorm & $\begin{array}{l}\text { Toud to } \\
\text { gond }\end{array}$ \\
\hline Imapt15.Jpg & 259 & s. & 35.26 & 397.13 & Tnodentores & \begin{tabular}{|l} 
Touch to \\
groud
\end{tabular} \\
\hline Image:16Jps & 3.76 & 13.13 & 24.25 & 53137 & $\begin{array}{c}\text { Severs } \\
\text { Tundtaborm }\end{array}$ & $\begin{array}{l}\text { Touch to } \\
\text { Foud }\end{array}$ \\
\hline Image: $17 \mathrm{Jps}$ & 138 & 8 & 66.28 & 323.76 & $\frac{\text { No }}{\text { Tundentoen }}$ & $\begin{array}{l}\text { Not } \\
\text { Toud to } \\
\text { growd }\end{array}$ \\
\hline Imaget $18 . j p g$ & 259 & 95 & 35.24 & 384.47 & Tnedentom & $\begin{array}{l}\text { Touch to } \\
\text { grousd }\end{array}$ \\
\hline Imaget $9.5 p$ & 265 & 11.12 & 34.38 & 45003 & Tnedentores & $\begin{array}{l}\text { Touch to } \\
\text { groud }\end{array}$ \\
\hline Image20.Jpg & 194 & 1037 & 47.08 & 419.67 & Tnedentere & $\begin{array}{l}\text { Toud to } \\
\text { grousd }\end{array}$ \\
\hline Image21.Jpg & 398 & 15 & 2288 & 607.05 & \begin{tabular}{c|c|} 
Serent \\
Dindestorem
\end{tabular} & \begin{tabular}{|l} 
Toudu to \\
ground
\end{tabular} \\
\hline Image22Jpl & 3.19 & 9.25 & 28.55 & 384.63 & Thadentorm & \begin{tabular}{|l|} 
Touch to \\
ground
\end{tabular} \\
\hline Image23j.jpg & 221 & 95 & 41.24 & 384.47 & Tnetenter & $\begin{array}{l}\text { Touch to } \\
\text { groud }\end{array}$ \\
\hline Image24Jpg & 294 & 10.69 & 30.97 & 43262 & \begin{tabular}{|c|} 
Severe \\
Dundentuen
\end{tabular} & \begin{tabular}{|l|} 
Tount to \\
gyous
\end{tabular} \\
\hline Image25.Jpg & 234 & 9.13 & 38.93 & 389.45 & Tindentern & $\begin{array}{l}\text { Touch to } \\
\text { ground }\end{array}$ \\
\hline $\operatorname{lng} 25.5 p$ & 301 & 10.87 & 30.27 & 43991 & \begin{tabular}{|c|} 
Sever \\
Tumdentome
\end{tabular} & \begin{tabular}{|l|} 
Touch to \\
groud
\end{tabular} \\
\hline Image:27.jpg & 253 & 10.5 & 36.05 & 42494 & Tyedentem & $\begin{array}{l}\text { Tood to } \\
\text { groud }\end{array}$ \\
\hline Image28Jpg & 2.1 & 9.66 & 43.48 & 38634 & Tnisenower & $\begin{array}{l}\text { Touch to } \\
\text { grousd } \\
\text { Yose }\end{array}$ \\
\hline Image $29 . \mathrm{Jpg}$ & 1.65 & 9.38 & 55.17 & 38124 & Thedenwern & $\begin{array}{l}\text { Nouct to } \\
\text { Groved }\end{array}$ \\
\hline Image $30 \mathrm{Jpg}$ & 186 & 8.94 & 90.05 & 394.71 & Tundentere & $\begin{array}{l}\text { Touch to } \\
\text { Froud }\end{array}$ \\
\hline
\end{tabular}

\begin{tabular}{|c|c|c|c|c|c|c|}
\hline Image $31 \mathrm{Jpg}$ & 2.39 & 9.63 & 38.18 & 389.52 & Thunderstorm & $\begin{array}{l}\text { Touch to } \\
\text { ground }\end{array}$ \\
\hline Image $32 \mathrm{Jpg}$ & 2.5 & 10.31 & 36.43 & 417.25 & Thunderstorm & $\begin{array}{l}\text { Touch to } \\
\text { ground }\end{array}$ \\
\hline Image 33Jpg & 2.68 & 9.75 & 33.98 & 394.58 & Thunderstorm & $\begin{array}{l}\text { Touch to } \\
\text { ground }\end{array}$ \\
\hline Image 34Jpg & 1.94 & 9.63 & 47.05 & 389.52 & Thunderstorm & $\begin{array}{l}\text { Touch to } \\
\text { ground }\end{array}$ \\
\hline Image $35 \mathrm{Jpg}$ & 2.3 & 9.13 & 39.68 & 431.65 & $\begin{array}{c}\text { Sever } \\
\text { Thunderstorm }\end{array}$ & $\begin{array}{l}\text { Touch to } \\
\text { ground }\end{array}$ \\
\hline Image36Jpg & 3.27 & 11 & 27.9 & 445.17 & $\begin{array}{c}\text { Sever } \\
\text { Thunderstorm }\end{array}$ & $\begin{array}{l}\text { Touch to } \\
\text { ground }\end{array}$ \\
\hline Image $37 \mathrm{Jpg}$ & 2.19 & 9.13 & 41.71 & 392.43 & Thunderstorm & $\begin{array}{l}\text { Touch to } \\
\text { ground }\end{array}$ \\
\hline Image38Jpg & 2.12 & 9.6 & 43.01 & 388.63 & Thunderstorm & $\begin{array}{l}\text { Touch to } \\
\text { ground }\end{array}$ \\
\hline Image39.Jpg & 3.36 & 10.88 & 27.12 & 372.92 & Thunderstorm & $\begin{array}{l}\text { Not } \\
\text { Touch to } \\
\text { ground }\end{array}$ \\
\hline Image 40 Jpg & 2.9 & 15.13 & 31.43 & 543.62 & $\begin{array}{c}\text { Sever } \\
\text { Thunderstorm }\end{array}$ & $\begin{array}{l}\text { Touch to } \\
\text { ground }\end{array}$ \\
\hline Image $41 \mathrm{Jpg}$ & 2.85 & 12.88 & 31.98 & 462.78 & $\begin{array}{c}\text { Sever } \\
\text { Thunderstorm }\end{array}$ & $\begin{array}{l}\text { Touch to } \\
\text { ground }\end{array}$ \\
\hline Image42Jpg & 3.2 & 12.25 & 28.48 & 464.23 & $\begin{array}{c}\text { Sever } \\
\text { Thunderstorm }\end{array}$ & $\begin{array}{l}\text { Touch to } \\
\text { ground }\end{array}$ \\
\hline Image43.Jpg & 3.63 & 14.25 & 25.11 & 512 & $\begin{array}{c}\text { Sever } \\
\text { Thunderstorm }\end{array}$ & $\begin{array}{l}\text { Touch to } \\
\text { ground }\end{array}$ \\
\hline Image44.Jpg & 2.12 & 8.75 & 42.99 & 314.39 & $\begin{array}{c}\text { No } \\
\text { Thunderstorm }\end{array}$ & $\begin{array}{l}\text { Not } \\
\text { Touch to } \\
\text { ground }\end{array}$ \\
\hline Image $45 \mathrm{Jpg}$ & 2.71 & 10.75 & 33.63 & 378.45 & Thunderstorm & $\begin{array}{l}\text { Touch to } \\
\text { ground }\end{array}$ \\
\hline Image46Jpg & 2.59 & 9.88 & 35.19 & 354.81 & Thunderstorm & $\begin{array}{l}\text { Touch to } \\
\text { ground }\end{array}$ \\
\hline Image47.Jpg & 2.63 & 10.31 & 34.65 & 380.44 & Thunderstorm & $\begin{array}{l}\text { Touch to } \\
\text { ground }\end{array}$ \\
\hline Image48.Jpg & 2.71 & 12.25 & 33.63 & 440.14 & $\begin{array}{c}\text { Severe } \\
\text { Thunderstorm }\end{array}$ & $\begin{array}{l}\text { Touch to } \\
\text { ground }\end{array}$ \\
\hline Image49.Jpg & 2.06 & 13.25 & 44.24 & 486.07 & $\begin{array}{c}\text { Severe } \\
\text { Thunderstorm }\end{array}$ & $\begin{array}{l}\text { Touch to } \\
\text { ground }\end{array}$ \\
\hline Image 50 Jpg & 2.17 & 9.6 & 42 & 344.93 & $\begin{array}{c}\text { Severe } \\
\text { Thunderstorm }\end{array}$ & $\begin{array}{l}\text { Touch to } \\
\text { ground }\end{array}$ \\
\hline Image $51 \mathrm{Jpg}$ & 332 & 13.3 & 27.45 & 485.23 & $\begin{array}{c}\text { Severe } \\
\text { Thunderstorm }\end{array}$ & $\begin{array}{l}\text { Touch to } \\
\text { ground }\end{array}$ \\
\hline Image 52Jpg & 1.84 & 9.8 & 49.53 & 352.11 & Thunderstom & $\begin{array}{l}\text { Touch to } \\
\text { ground }\end{array}$ \\
\hline Image $53 \mathrm{Jpg}$ & 2.4 & 9.9 & 37.97 & 355.71 & Thunderstorm & $\begin{array}{l}\text { Touch to } \\
\text { ground }\end{array}$ \\
\hline Image54Jpg & 1.9 & 10.38 & 47.97 & 372.95 & Thunderstorm & $\begin{array}{l}\text { Touch to } \\
\text { ground }\end{array}$ \\
\hline Image $55 \mathrm{Jpg}$ & 2.86 & 13.12 & 31.87 & 491.4 & $\begin{array}{c}\text { Severe } \\
\text { Thunderstorm }\end{array}$ & $\begin{array}{l}\text { Touch to } \\
\text { ground }\end{array}$ \\
\hline Image 56 Jpg & 2.32 & 9.06 & 39.28 & 325.53 & Thunderstorm & $\begin{array}{l}\text { Touch to } \\
\text { ground }\end{array}$ \\
\hline Image 57.Jpg & 1.94 & 9.25 & 46.98 & 332.35 & $\begin{array}{c}\text { No } \\
\text { Thunderstorm }\end{array}$ & $\begin{array}{l}\text { Not } \\
\text { Touch to } \\
\text { ground }\end{array}$ \\
\hline Image 58 Jpg & 2.67 & 9.8 & 34.13 & 352.11 & Thunderstorm & $\begin{array}{l}\text { Touch to } \\
\text { ground }\end{array}$ \\
\hline Image59.Jpg & 1.8 & 9.8 & 50.63 & 352.11 & Thunderstorm & $\begin{array}{l}\text { Touch to } \\
\text { ground }\end{array}$ \\
\hline
\end{tabular}

\section{Volume 6 Issue 7, July 2017 www.ijsr.net}

Licensed Under Creative Commons Attribution CC BY 


\section{International Journal of Science and Research (IJSR) ISSN (Online): 2319-7064}

Index Copernicus Value (2015): 78.96 | Impact Factor (2015): 6.391

\begin{tabular}{|c|c|c|c|c|c|c|}
\hline Imag660.jpg & 256 & 9.25 & 35.6 & 33235 & $\begin{array}{c}\mathrm{N}_{0} \\
\text { Thamberstoen }\end{array}$ & $\begin{array}{l}\text { Noc } \\
\text { Touch to } \\
\text { ground }\end{array}$ \\
\hline Image6 $1 . j p g$ & 181 & 875 & 30.35 & 31439 & $\frac{\mathrm{Ni}}{\text { Tindestora }}$ & $\begin{array}{l}\text { Nok } \\
\text { Touch to } \\
\text { goroud }\end{array}$ \\
\hline Image6 $2.5 p g$ & 222 & 9.18 & 41.05 & 32984 & $\begin{array}{c}\mathrm{No}_{0} \\
\text { Thintestora }\end{array}$ & $\begin{array}{l}\text { Noc } \\
\text { Touch to } \\
\text { goved }\end{array}$ \\
\hline Image6 $3 . \mathrm{Jpg}$ & 251 & 9.37 & 36.31 & 336.66 & $\begin{array}{c}\mathrm{No}_{0} \\
\text { Thistestorat }\end{array}$ & $\begin{array}{l}\text { Nor } \\
\text { Touch to } \\
\text { ground }\end{array}$ \\
\hline Imag6 $64 . j p g$ & 2.4 & 9.75 & 37.97 & 35032 & Tundestom & $\begin{array}{l}\text { Nok } \\
\text { Touch to } \\
\text { grousd }\end{array}$ \\
\hline Image65.Jpg & 271 & 9.86 & 33.63 & 35427 & Thundestoca & $\begin{array}{l}\text { Touch to } \\
\text { ground }\end{array}$ \\
\hline
\end{tabular}

In table 3, it calculate wavelength of each input image by standard formula and result of thunderstorm is depend upon range of wavelength. If wavelength lies between the range $350-450$ then results is thunderstorm otherwise there is no thunderstorm. Also it finds out weather the lightning touches to the ground or not.

Table 4: Prediction of Thunderstorm

\begin{tabular}{|c|c|c|c|}
\hline Image Number & $\begin{array}{l}\text { Experimentally } \\
\text { Obtained Result }\end{array}$ & \begin{tabular}{|c|} 
Historically \\
Established Result
\end{tabular} & Prediction \\
\hline Image1.Jpg & Thunderstorm & Thunderstorm & TRUE \\
\hline Image2.Jpg & Thunderstorm & Thunderstorm & TRUE \\
\hline Image3.Jpg & Thunderstorm & Thunderstorm & TRUE \\
\hline Image4.Jpg & Thunderstorm & Thunderstorm & TRUE \\
\hline Image5.Jpg & Thunderstorm & Thunderstorm & TRUE \\
\hline Image6.Jpg & Thunderstorm & Thunderstorm & TRUE \\
\hline Image7.Jpg & Thunderstorm & Thunderstorm & TRUE \\
\hline Image8.Jpg & Thunderstorm & Thunderstorm & TRUE \\
\hline Image9.Jpg & Thunderstorm & Thunderstorm & TRUE \\
\hline Image10.Jpg & Thunderstorm & No Thunderstorm & FALSE \\
\hline Image11.Jpg & Thunderstorm & Thunderstorm & TRUE \\
\hline Image12.Jpg & Thunderstorm & Thunderstorm & TRUE \\
\hline Image13.Jpg & Thunderstorm & Thunderstorm & TRUE \\
\hline Image14.Jpg & Thunderstorm & Thunderstorm & TRUE \\
\hline Image15.Jpg & Thunderstorm & Thunderstorm & TRUE \\
\hline Image16.Jpg & Thunderstorm & Thunderstorm & TRUE \\
\hline Image17.Jpg & No Thunderstorm & No Thunderstorm & FALSE \\
\hline Image18.Jpg & Thunderstorm & \begin{tabular}{|l|} 
Thunderstorm \\
\end{tabular} & TRUE \\
\hline Image19.Jpg & Thunderstorm & Thunderstorm & TRUE \\
\hline Image20.Jpg & Thunderstorm & Thunderstorm & TRUE \\
\hline Image21.Jpg & Thunderstorm & Thunderstorm & TRUE \\
\hline Image22.Jpg & Thunc & Thunderstorm & TRUE \\
\hline Image23.Jpg & Thunderstorm & Thunderstorm & TRUE \\
\hline Image24.Jpg & Thunderstorm & Thunderstorm & TRUE \\
\hline Image25.Jpg & Thunderstorm & Thunderstorm & TRUE \\
\hline Image 26.Jpg & Thunderstorm & Thunderstorm & TRUE \\
\hline Image27.Jpg & Thunderstorm & Thunderstorm & TRUE \\
\hline Image28.Jpg & Thunderstorm & Thunderstorm & TRUE \\
\hline Image29.Jpg & Thunderstorm & Thunderstorm & TRUE \\
\hline Image30.Jpg & Thunderstorm & Thunderstorm & TRUE \\
\hline Image31.Jpg & Thunderstorm & Thunderstorm & TRUE \\
\hline Image32.Jpg & Thunderstorm & Thunderstorm & TRUE \\
\hline Image33.Jpg & Thunderstorm & Thunderstorm & TRUE \\
\hline Image34.Jpg & Thunderstorm & Thunderstorm & TRUE \\
\hline Image35.Jpg & Thunderstorm & Thunderstorm & TRUE \\
\hline Image36.Jpg & Thunderstorm & Thunderstorm & TRUE \\
\hline Image37.Jpg & Thunderstorm & Thunderstorm & TRUE \\
\hline Image38.Jpg & Thunderstorm & Thunderstorm & TRUE \\
\hline Image39.Jpg & Thunderstorm & Thunderstorm & TRUE \\
\hline Image40.Jpg & Thunderstorm & Thunderstorm & TRUE \\
\hline
\end{tabular}

\begin{tabular}{|l|c|c|c|}
\hline Image41.Jpg & Thunderstorm & Thunderstorm & TRUE \\
\hline Image42.Jpg & Thunderstorm & Thunderstorm & TRUE \\
\hline Image43.Jpg & Thunderstorm & Thunderstorm & TRUE \\
\hline Image44.Jpg & No Thunderstorm & No Thunderstorm & FALSE \\
\hline Image45.Jpg & Thunderstorm & Thunderstorm & TRUE \\
\hline Image46.Jpg & Thunderstorm & Thunderstorm & TRUE \\
\hline Image47.Jpg & Thunderstorm & Thunderstorm & TRUE \\
\hline Image48.Jpg & Thunderstorm & Thunderstorm & TRUE \\
\hline Image49.Jpg & Thunderstorm & Thunderstorm & TRUE \\
\hline Image50.Jpg & Thunderstorm & Thunderstorm & TRUE \\
\hline Image51.Jpg & Thunderstorm & Thunderstorm & TRUE \\
\hline Image52.Jpg & Thunderstorm & Thunderstorm & TRUE \\
\hline Image53.Jpg & Thunderstorm & Thunderstorm & TRUE \\
\hline Image54.Jpg & Thunderstorm & Thunderstorm & TRUE \\
\hline Image55.Jpg & Thunderstorm & Thunderstorm & TRUE \\
\hline Image56.Jpg & Thunderstorm & Thunderstorm & TRUE \\
\hline Image57.Jpg & No Thunderstorm & No Thunderstorm & FALSE \\
\hline Image58.Jpg & Thunderstorm & Thunderstorm & TRUE \\
\hline Image59.Jpg & Thunderstorm & Thunderstorm & TRUE \\
\hline Image60.Jpg & No Thunderstorm & No Thunderstorm & FALSE \\
\hline Image61.Jpg & No Thunderstorm & No Thunderstorm & FALSE \\
\hline Image62.Jpg & No Thunderstorm & No Thunderstorm & FALSE \\
\hline Image63.Jpg & No Thunderstorm & No Thunderstorm & FALSE \\
\hline
\end{tabular}

Table 4 shows prediction of thunderstorm in which comparison of historical result and experimentally obtained result (proposed system result) and calculate prediction Prediction is calculated, if both are having thunderstorm then prediction is true if one of the result is no thunderstorm then result is false. There it is observed that the proposed method predicts the thunderstorms with an average accuracy of $89.2 \%$ which is far better than the existing system.

\section{Conclusions}

In the proposed system, experiments have been conducted with k-medoids clustering technique and Haar wavelet transforms for the prediction of thunderstorms. It was demonstrated that the proposed system gives better result as compare to the previous methods such as STP model, MOM model, CG model, LM model, QKP model, DBD model in the detection of thunderstorms. In order to compute accuracy, the four basic performance measures is considered i.e. sensitivity, specificity, accuracy and precision are computed. The proposed method predicts the thunderstorms with an average accuracy of $89.2 \%$ which is far better than the existing system. In this we discussed about the prediction of thunderstorms and lightning detection system. The proposed system uses hybrid approach, this system adopted clustering and wavelet transform techniques for thunderstorms and lightning detection using image processing and detecting whether lightning produce from thunderstorm touches to the ground or not.

\section{References}

[1] Himadri Chakrabarty, Murthy, C. A., Sonia Bhattacharya and Ashis Das Gupta, "Application of Artificial Neural Network to Predict SquallThunderstorms Using RAWIND Data," International Journal of Scientific and Engineering Research, 2013, pp. 1313-1318.

[2] Litta, A.J., Sumam Mary Idicula and Naveen Francis C, "Artificial Neural Network Model for the Prediction of 


\section{International Journal of Science and Research (IJSR) \\ ISSN (Online): 2319-7064 \\ Index Copernicus Value (2015): 78.96 | Impact Factor (2015): 6.391}

Thunderstorms over Kolkata", International Journal of Computer Applications, 2012, pp.50-55.

[3] Rudolf kaltenbock, Gerhard Diendorfer and Nikolai Dotzek, "Evaluation of Thunderstorm Indices from ECMWF Analyses, Lightning data and Severe Storm reports," Atmospheric research Journal, Elsevier, 2009, pp. 381-396.

[4] Tajbakhsh, S., Ghafarian, P, and Sahraian, F., "Instability Indices and Forecasting Thunderstorms: the case of 30 April 2009," Natural hazards and Earth System Sciences, 2012, pp. 403-413.

[5] Mahesh Anand, s., Ansupa Dashi, Jagadeesh Kumar, Amit Kesarkar, "Prediction and Classification of Thunderstorms using Artificial Neural Network," International journal of Engineering, Science and Technology, 2011, pp. 4031-4035.

[6] Alwin Haklander, Aarnout Van Delden, "Thunderstorm Predictors and their Forecast Skill for the Netherlands," Atmospheric Research, Elsevier, 2003, pp. 273-299.

[7] Pinto, "The Sensitivity of The Thunderstorm Activity in the city of Sao paulo to temperature Changes: predicting the Future Activity for Different Scenarios," International Lightning Detection Conference, 2012, pp. 1-4.

[8] Alan czarnetz ki, C., "Evaluation of a Forecast strategy for Nocturnal Thunderstorms that Produce heavy rain," pp. 25-31.

[9] C. Fraley and A. Raftery, "How Many Clusters? Which Clustering method? Answers via Model-Based Cluster Analysis," The Computer Journal, 1998, pp. 578-588.

[10] Candra Dewi, Mega Satya Ciptaningrun, Nmuh Arif Rahman,Denoising Cloud Interference on Landsat Satellite Image Using Discrete Haar Wavelet Transformation," International Journal of Computer Science and Information Security, 2012, pp.27-31.

[11] Kanwaljot Singh Sidhu, Baljeet Singh Khaira, Ishpreet Singh Virk,"Medical Image Denoising In The Wavelet Domain Using Haar and DB3 Filtering," International Refereed Journal of Engineering and Science, 2012, pp. 1-8.

[12] Anisha Ravinder, P., Prudhvi Kumar Reddy, K., Narasimha Prasad L.V., "Detection of Wavelengths for Hail Identification Using Satellite Imagery of Clouds," IEEE CICSYN, 2013, pp. $205-211$.

[13] David Bright, R., Matthew Wandishin, S., Ryan Jewell, E., and Steven Weiss, "A Physically Based Parameter for Lightning Prediction and its Calibration in Ensemble Forecasts," Conference on Meteorological Applications of Lightning Data, AMS, 2005, pp. 1-11.

\section{Author Profile}

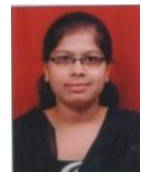

Rasika V.kalbende has Perusing M.Tech degree in Computer Science and Engineering from G.H. Raisoni college of Engineering Amravati. She is now M-Tech student .She has published 1 research paper in reputed international journal. Her area of research includes image processing

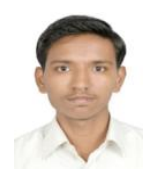

Prof. Nitin. A. Shelke has received M.Tech degree in Computer Science and Engineering from Government College of Engineering Amravati. He is currently working as an Assistant Professor in G. H. Raisoni College of Engineering and Management Amravati,
Maharashtra, India. He has published the 12 research paper in reputed International Journal. His area of research includes Image Processing, Soft Computing and Genetic Algorithm. 